\title{
Función instrumental de la segunda lengua y educación bilingüie
}

\author{
Minnie Lozada T. \\ Universidad Nacional Mayor de San Marcos \\ lozadame@speedy.com.pe
}

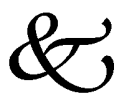

\begin{abstract}
Resumen
Aunque se entiende que en la educación bilingüe intercultural la enseñanza aprendizaje de la segunda lengua cumple una función instrumental junto a la lengua materna, no se tiene conocimiento que esto se esté llevando a cabo en nuestro país. La autora plantea que esto se debe, en parte, a que se están confundiendo nociones básicas, entre ellas, lo que es el aprendizaje de contenidos del currículo en segunda lengua con el que se realiza en lengua materna, $y$, el de dominio del lenguaje para fines académicos con el de lenguaje social. Para contribuir a esclarecer estos puntos, el presente artículo estudia el aspecto linguiístico de tres programas educativos que, desde diferentes perspectivas, buscan desarrollar el uso académico de la segunda lengua.
\end{abstract}

Palabras claves: Educación bilinguie, Lenguaje académico, Segunda lengua.

\begin{abstract}
Instrumental function of a second language and bilingual education

Even though in bilingual intercultural education the learning of a second language should fulfill an instrumental function together with the mother tongue, we have no information that this is being carried out in our country. The author states that this is, partly, due to a confusion of certain basic notions, among them, the learning of content in a first and in a second language, and what it means to master a language for academic purposes. To help clarify these points, the author studies three programs which, from different perspectives, have as an objective the development of academic language.
\end{abstract}

Key words: Bilingual education, Academic language, Second language. 


\section{Introducción}

La educación intercultural, modalidad que tratamos de desarrollar en el país, puede incluir, entre sus componentes, la adquisición de una segunda lengua (L2) y su uso al lado de la lengua materna (L1). Es dentro de este tema de la linguística aplicada que se ubica el objeto del presente artículo. Lo que nos interesa es, específicamente, la segunda lengua en su función instrumental, esto es, en su uso dentro del proceso de enseñanza aprendizaje de contenidos del currículo.

El componente de segunda lengua puede tratarse de distintas maneras, una, mediante un curso especial de L2; otra, través de las diferentes líneas del currículo. Tradicionalmente, el profesor de lenguaje ha sido considerado el único responsable del desarrollo de la lengua en la escuela, por ello, en las demás líneas, los otros docentes no contribuían, y algunos aún no contribuyen, con esta labor. Planteamos que el enfoque del lenguaje a través del currículo se debe tener en cuenta, no sólo para la lengua materna, sino también para la segunda lengua en situaciones de educación bilinguie intercultural (EBI), ya que en ella la lengua cumple la función instrumental antes mencionada.

Téngase presente que si los profesores de las distintas áreas del currículo (Sociales, Naturales, Físico Matemáticas, etc.) no consideran parte de su responsabilidad el prestarle atención a la lengua materna del alumno, cuanto más difícil será que lo hagan con la segunda lengua en los programas de educación intercultural. Por medio de evaluaciones internacionales, y de los mismos maestros, conocemos que hay dificultades con el uso del lenguaje en nuestras escuelas. Consideremos la información de Dante Bermeo Córdova, un profesor de secundaria que participa en un curso de especialización que ofrece el Ministerio de Educación, actualmente, en coordinación con la Universidad Católica de Lima. En un trabajo que le solicitamos, al analizar el dominio de habilidades de lengua materna castellana (L1) que tienen 18 de sus 20 alumnos de Primero de Secundaria, el docente observa que frente a un $90 \%$ (18 alumnos) que dominan el castellano oral, sólo un $65 \%$ tiene control también de la habilidad de comprensión de lectura. Ante situaciones como ésta, surge la interrogante de cómo pueden estudiar los que forman ese $35 \%$ que no puede leer. 
Tabla 1. Indicadores de habilidades lingüísiticas

\begin{tabular}{lcc}
\hline \multicolumn{1}{c}{ HABILIDADES LINGÜISTICAS } & $\begin{array}{c}\text { Número de } \\
\text { estudiantes }\end{array}$ & $\%$ \\
\hline $\begin{array}{l}\text { Comprensión oral, comprensión lecto- } \\
\text { ra, expresión oral y expresión escrita }\end{array}$ & 05 & $25 \%$ \\
$\begin{array}{l}\text { Comprensión oral, compresión lectora y } \\
\text { expresión oral. }\end{array}$ & 13 & $65 \%$ \\
\begin{tabular}{l} 
Comprensión oral y expresión oral. \\
\hline
\end{tabular}
\end{tabular}

Centro ESMED Ishichihui - Región San Martín, 2007.

Si con la lengua materna, en la escuela hay dificultades como la arriba señalada, ¿cómo hacer para que en la educación bilinguie intercultural se desarrolle también la segunda lengua, y, por medio de ésta, se aprendan contenidos del currículo, esto es, conocimiento nuevo y desarrollo de capacidades? Buscando respuestas, proyectamos una revisión de experiencias educativas que se desarrollan en contextos diversos para ver como se trabaja la L2 en la escuela para fines académicos. Dejamos constancia que entendemos que la educación se debe hacer en las dos lenguas, pero nuestro interés, en este artículo, es la lengua aprendida como L2, no como L1.

\section{Marco conceptual}

En lo que se refiere al empleo de dos lenguas en la educación, concordamos con Baker, cuando citando a Cazden y Snow señala que "La enseñanza bilinguie es una simple etiqueta para un fenómeno complejo". (1997: 217) Si bien muchos estamos de acuerdo con la inclusión de un componente bilinguie en la educación, somos concientes que la manera como se entiende la presencia de las dos lenguas en ésta, varía según la orientación de las personas. Nos parece razonable también la aclaración de Baker en el sentido de que "la enseñanza bilinguie no se ocupa necesariamente del empleo equilibrado de dos lenguas en la clase. Tras la enseñanza bilinguie están diversas y conflictivas ideologías de para qué es la enseñanza” (Baker, 1997: 219). Esto explica, en parte, la variedad en el peso que se otorga a cada una de las dos lenguas de la educación bilinguie. Pensamos que un primer objetivo debe ser que el alumno alcance un dominio de habilidades orales y escritas en la L2 
para poder realizar sus estudios. La pregunta es, dadas las características de nuestra escuela, ¿cómo ayudar a este proceso de avance en el control de la lengua?

Debemos precisar que entendemos el término 'segunda lengua', para efectos de esta presentación, en el sentido de aquella lengua que se adquiere o aprende después de la primera o materna. En este escrito, vamos a centrar nuestra atención en la adquisición o aprendizaje de ésta dentro de un contexto particular, el de la escuela. Como en el Perú el castellano es segunda lengua en varias zonas, nos referimos a esta lengua aunque sabemos que todas las lenguas pueden ser aprendidas o adquiridas como L2.

Si deseamos que los estudiantes desarrollen habilidades orales y escritas de L2 para fines académicos o de estudio, es necesario aclarar lo que queremos decir cuando nos referimos al dominio o suficiencia que se espera lograr en esta lengua. Aunque hay diferentes maneras de conceptualizar la suficiencia en segunda lengua, concordamos con lo que Cummins (2002) plantea al respecto. Según este autor, el objetivo no es que el alumno que adquiere lengua logre el nivel de un hablante nativo. De ahí que, en el caso de la educación bilingüe, lo que debemos buscar es un dominio de la segunda lengua que le permita al estudiante usarla de manera adecuada en el contexto de la escuela. Al respecto, "el constructo del dominio académico del idioma no alude a una idea absoluta del dominio de uso del idioma, sino al grado en el que una persona tiene acceso y maestría suficiente para comprender y utilizar el tipo concreto de lenguaje empleado en contextos educativos necesario para cumplimentar las tareas académicas". (Cummins, 2002: 83-84)

Los que tienen la capacidad de orientar la educación bilinguie intercultural en nuestro país deberán plantearse, previo análisis y evaluación del desempeño linguiístico de los alumnos en programas piloto, cuáles pueden ser los grados de dominio de segunda lengua que podemos establecer, realistamente, como meta, teniendo en cuenta los distintos contextos sociolinguiísticos de los alumnos. Podemos comenzar, quizá, planteándonos unos esenciales mínimos, los cuales se irán ajustando a medida que adquirimos mayor conocimiento sobre lo que sucede en las escuelas, no con la enseñanza, sino con el aprendizaje del castellano como L2. 


\section{Antecedentes de la enseñanza aprendizaje en la L2}

\subsection{La educación tradicional}

Recordemos que la educación que se ofrecía en el pasado se hacía en castellano, no importaba cuál fuera la lengua materna del niño, ni si las pautas que daban las autoridades y llevaban a la práctica los docentes eran pertinentes para los diferentes contextos socioculturales. Conocemos los resultados negativos de esa educación y las consecuencias que ésta tuvo en los alumnos. Entre ellas, una de las que destaca es la falta de desarrollo del sentimiento de identidad de los alumnos debido a que se buscó asimilarlos a modelos que les eran extraños, disminuyendo el sentido de pertenencia a su cultura, y su autoestima. Otra consecuencia fue la dificultad de los alumnos para lograr un adecuado rendimiento escolar. Como lo usual era que el estudio y las actividades en la escuela se desarrollaran en castellano, los estudiantes hablantes de otras lenguas no entendían bien lo que se pretendía enseñarles en esta lengua, y lo más grave era que ni las autoridades educativas ni los docentes eran concientes de la situación. No se pensaba ni en la necesidad de un aprendizaje previo de la L2 ni en un refuerzo o desarrollo mayor de ella a medida que se avanzaba en la escuela Era natural que como resultado de la situación, los estudiantes tuvieran dificultad para la adquisición de conocimientos y para el desarrollo de distintas capacidades y de actitudes positivas hacia lo suyo. La dificultad es que esta situación continua en muchas escuelas. Al respecto, veamos lo que piensa el profesor R. Chuquimamani (Ministerio de Educación, 2005):

En la práctica muchos docentes de aulas EBI trabajan temas correspondientes a las diferentes áreas de desarrollo en castellano, pero no enseñan el castellano. La confusión parte del hecho de comprender por igual enseñar en castellano con enseñar el castellano.

Enseñar en castellano implica utilizar la lengua castellana como instrumento de comunicación. Esto supone que el aprendiz ya maneja la lengua instrumental y lo que aprende en ese momento es otro contenido, fuera de lo linguístico.

Enseñar el castellano, en cambio, implica que el objeto de nuestro estudio es la propia lengua .... (Ministerio de Educación, 2005: A manera de prólogo) 


\subsection{Alternativas de solución a las dificultades lingüísticas en el colegio}

La educación intercultural bilingüe es una alternativa de enseñanza aprendizaje que se está aplicando para mejorar la formación en las escuelas. Si bien esta modalidad ha abierto un espacio a la cultura y a la lengua originaria de los estudiantes, también, con esta educación, se busca resolver la dificultad que pueden tener los estudiantes cuando necesitan usar la segunda lengua en la escuela. Para ello, se ha previsto que parte del componente de segunda lengua, el castellano, comience con la enseñanza aprendizaje de dicha lengua, teniendo en cuenta distintos contextos sociolinguiísticos. Una vez que se tenga una base, se empleará dicha lengua para el aprendizaje de contenidos del currículo en ella. Este planteamiento es diferente al de los programas llamados de inmersión, en los cuales el alumno desde el primer día de clase se mueve en un contexto de segunda lengua.

Por medio de la información que se ha difundido hasta ahora, a través de los maestros, sobre el aspecto bilinguie de nuestra educación, sabemos que sí estamos ofreciendo al alumno enseñanza de la segunda lengua, el castellano, dentro del área de Comunicación. Se comienza con la práctica oral, para pasar luego a la lengua escrita incluyendo la enseñanza de aspectos estructurales y/o funcionales de la L2, los cuales pueden contribuir al desarrollo de habilidades como hablar, escuchar, leer y escribir. En algunas escuelas, también se intenta hace práctica con actividades comunicativas partiendo de temas vinculados a la cultura de los alumnos.

Sobre el punto que concentra nuestra atención, la enseñanza en castellano como L2 en nuestro país, hemos conseguido poca información. Una interrogante que se nos plantea es si hemos llegado a hacer enseñanza en L2. Sabemos que hay profesores que continúan con la enseñanza aprendizaje en castellano, pero como si esta lengua fuera la lengua materna de los estudiantes. Y no siempre es así, en muchos casos es la L2 de ellos. ¿Estamos usando en el país el castellano como L2 para aprender cursos como Naturales, Sociales, Matemática? No conocemos que esto se esté trabajando de manera sistemática actualmente. Entendemos la preocupación de los maestros de asegurarse que el 
alumno tenga una base de segunda lengua antes de hacer estudios en ella. Pero, no debemos olvidar que si queremos implementar una educación intercultural que sea bilinguie, debemos dar a los alumnos, al lado de la lengua materna, la oportunidad del aprendizaje, también, de y en la segunda lengua. Hay pocas propuestas en nuestro medio que han difundido cómo realizar esta enseñanza en la L2, y, de ahí, este estudio de algunas experiencias educativas, tanto peruanas como de fuera. El recuerdo del pasado puede hacernos sentir un cierto temor de enseñar ahora en la L2, pero debemos tener en cuenta que esto depende de cómo se entiende lo que es enseñar en una segunda lengua. Consideramos que enseñar en una L2 no es igual que enseñar en una L1.

\section{Materiales estudiados}

\subsection{La segunda lengua para fines de estudio}

Las fuentes empleadas son estudios sobre experiencias y textos de enseñanza en las que la segunda lengua se emplea para fines académicos. Consideramos en primer lugar la investigación de J. Cummins, un especialista del Canadá. Luego revisamos los informes de tres experiencias de educación: la primera tiene que ver con material de Ciencias Naturales del Programa Experimental de Educación Bilinguie de Puno (Ministerio de Educación: 1989, 2005), la segunda es una obra sobre el programa educativo CALLA o Cognitive Academic Language Learning Approach (Chamot y O’Malley, 1994) para la enseñanza de niños inmigrantes en los Estados Unidos, y finalmente, nos referimos a la experiencia peruana de inglés intensivo en la primaria del CEP Rosa de América (2007), en la que se hace lectura complementaria en segunda lengua para ciertos cursos del currículo, experiencia en la que hemos participado.

Ya en las dos últimas décadas del siglo pasado, estudios como los de Cummins (1986: 139-140) contribuyen a la precisión de ciertos conceptos. Dicho especialista observó que cuando los psicólogos y profesores, al realizar evaluaciones, incurrían en error respecto del rendimiento de sus alumnos en la escuela, esto se producía debido a la confusión teórica sobre la relación dominio de lengua y aprendizaje. Hay varios aspectos vinculados que faltaba investigar, entre ellos, la relación entre 
desempeño en lengua oral, lectura y otras habilidades académicas; el papel de un déficit linguiístico en las discapacidades de aprendizaje; y, la relación entre destrezas comunicativas de L2 en situación de interacción personal, y destrezas académicas en L2.

Con el tiempo, Cummins (2002), al continuar sus investigaciones sobre inmigrantes que se educan en la segunda lengua, el inglés, en el Canadá, vuelve sobre los conceptos antes mencionados deteniéndose en la distinción entre destrezas de lengua. La denominación que ahora emplea es destrezas conversacionales (o fluidez conversacional) y destrezas académicas. Para nuestro objetivo en este artículo es necesario considerar esta diferencia, en otras palabras, la que existe entre el dominio de la segunda lengua para situaciones de interacción frente a frente en la vida cotidiana, y la del dominio en el desempeño de tareas cognitivo / académicas.

\subsection{Destrezas académicas y tiempo de adquisición de la segunda lengua}

Ya en 1986 Cummins se refería a los resultados del trabajo de Saville y Troike en los que ella señalaba que "hay una diferencia cualitativa entre las destrezas y tácticas comunicativas que los niños encuentran efectivas para satisfacer sus necesidades y alcanzar sus objetivos sociales, y aquellos que son necesarios para un rendimiento académico escolar exitoso" (citado en Cummins 1986: 211). En relación con las destrezas académicas, la especialista llegó a la conclusión que tanto para grupos de inmigrantes que aprendían inglés como L2, como para los alumnos de programas de inmersión francesa, éstas destrezas tomaban entre cinco y siete años en desarrollarse.

También Cummins observa en el 2002, al analizar investigaciones realizadas en los EE.UU. y en Israel, en las cuales la segunda lengua era el inglés y el hebreo respectivamente, que el dominio de destrezas conversacionales se logra en menos tiempo que el de destrezas académicas. La explicación sobre esta diferencia en el tiempo parece deberse a que en el caso de las destrezas conversacionales

para desenvolverse de manera adecuada en situaciones comunicativas interpersonales suele hacer falta un conocimiento del idioma considera- 
blemente menor que el necesario en situaciones académicas. Las expectativas sociales del aprendiz y la sensibilidad a las claves contextuales e interpersonales (por ej., contacto visual, expresión facial, entonación, etc.) facilitan mucho la comunicación del sentido. (Cummins, 2002: 51)

En cambio, al referirse al desarrollo de las destrezas académicas, parte de las cuales requieren el uso de textos escritos, lo que sucede es que las claves sociales arriba señaladas,

están en gran medida ausentes de la mayoría de las situaciones académicas, que dependen del conocimiento del lenguaje para realizar las tareas de manera satisfactoria. En comparación con la conversación interpersonal, el lenguaje del texto suele incluir un vocabulario mucho menos frecuente, unas estructuras gramaticales complejas y mayores exigencias de memoria, análisis y otros procesos cognitivos. (Cummins, 2002: 51)

\section{Resultados de la revisión de experiencias en L2}

A continuación, revisemos aspectos de la enseñanza aprendizaje en la L2 en los programas que hemos seleccionado. Si bien estos son proyectos complejos, en este caso, nos centramos en el aspecto de la lengua académica. Comencemos por la experiencia del PEEB-Puno, para luego seguir con el programa CALLA, y finalmente, con el proyecto de inglés intensivo en Lima.

\subsection{Educación bilingüe quechua / aimara-castellano}

El Programa Experimental de Educación Bilinguie (PEEB-Puno), que inicia sus actividades en 1977, desarrolló una propuesta para la Primaria de la zona sur andina. Uno de sus aportes fue la preparación de textos para todas las líneas de acción educativa, tanto en lengua indígena como en castellano. Detengámonos en algunos planteamientos para la enseñanza aprendizaje en L2 que nos presenta este material. En la Guía metodológica Jakawisata (Ministerio de Educación, 1989) de Ciencias Naturales para sexto de Primaria, un aspecto que destaca es el cuidado que se ha tenido con la cultura de los alumnos. Así, en la lección sobre MEDICIONES EXACTAS se ofrece información sobre el concepto de medición en el área rural del altiplano señalando su diferencia con el de las áreas urbanas. Algunas declaraciones de personas encuestadas ilustran 
este pensamiento sobre medición: "Las personas no somos ni papa ni tela para pesarnos ni medirnos. La persona que se mide no crece más. Entonces ¿para qué medimos?" (INIDE, citado en Ministerio de Educación, 1989: 6b). La Guía también señala "que el problema sobre mediciones aparece cuando el campesino entra en contacto con las medidas oficiales. En este contexto los conceptos de medición y las unidades que se eligen en el campo resultan poco prácticas para la ciudad y no resulta fácil la comparación entre los dos sistemas”. (1989, 6 b)

En cuanto al lenguaje empleado en Jakawisata, el texto contiene algunas lecciones que se van a presentar a los niños en lengua materna y otras en castellano. Se hace notar al maestro que, a través del curso de Ciencias Naturales a la vez que los alumnos aprenden en su lengua originaria, también aprenden algo de castellano, que "el uso del castellano en la enseñanza de ciencias naturales refuerza el manejo de esta lengua que va adquiriendo el niño en la educación bilingiie". (Ministerio de Educación, 1989: XVIII) Como vemos, se espera que el profesor tome conciencia que en una línea diferente de la de Lenguaje o Comunicación, el alumno está también avanzando en su manejo de la segunda lengua. En este material se observa que no es necesario un dominio absoluto de la L2 para luego comenzar el aprendizaje en la L2. Se puede, una vez que se ha adquirido cierto conocimiento de la L2 continuar avanzando con el aprendizaje de y en la segunda lengua.

Por otro lado, la Guía destaca la importancia de distintas funciones del lenguaje, entre ellas una científica: "Esta unidad nos va a permitir reflexionar y tomar conciencia de la existencia de un lenguaje científico y un lenguaje poco preciso". (Ministerio de Educación, 1989: 6 b).

Entendemos que, en este caso, en que se trata el tema MEDICIONES EXACTAS, se busca que el alumno se ejercite en manejar la característica de precisión del lenguaje científico. Dos textos de Jakawisata, ejemplifican la búsqueda de la claridad y la precisión en la descripción. Obsérvese cómo entra la cantidad a través del empleo de los datos numéricos y el manejo de convenciones como las abreviaturas.

La comunidad es pequeña, tiene ganado y muchas familias.

Está cerca de la ciudad. En la escuela hay profesores y alumnos. 
Mi comunidad es pequeña. Está a $30 \mathrm{~km}$ de la ciudad de Puno. La comunidad tiene 250 familias que hacen un total de 1223 habitantes.

También tiene 500 cabezas de ganado. En la escuela hay

5 profesores y 140 alumnos.

Un punto importante en las orientaciones para usar este material son ciertos principios de interpretación de los textos que se consideran cuando se trabaja con cualquiera de las dos lenguas. Observemos, a continuación, un ejemplo de estos principios en relación con algunas aplicaciones en torno al terreno comunal:

- Ampliar las capacidades de observación y análisis de detalles. Ejemplo: ¿Cuánto mide el terreno comunal?,

- Incidir en el uso de la pregunta: ¿Por qué? Ejemplo: ¿Por qué hay tantos litigios por linderos de tierras?

- Relacionar un problema con ciertas causas y efectos. Ejemplo: ¿Será porque cada grupo utiliza maneras distintas para medir sus linderos?

- Proponer diferentes explicaciones y soluciones a un problema dado. Ejemplo: ¿O será que la delimitación fue hecha por alguien de una oficina que no conoce la historia de la comunidad?

- Admitir que las soluciones y explicaciones no son únicas. Hay diferentes maneras de pensar. Ejemplo: proponer una solución y debatirla, luego plantear otra solución.

Como vemos, con la práctica arriba sugerida se desarrolla en los alumnos habilidades como observar, analizar, identificar razones, relacionar causa y efecto, explicar y buscar soluciones, y entender que hay distintas alternativas de solución. Y junto con ellas, se va dando el desarrollo de las dos lenguas, primera y segunda, para expresar estas ideas.

A continuación, veamos otra manera de plantear la enseñanza aprendizaje en L2, la del programa CALLA, una experiencia extranjera.

\section{2 'CALLA', un modelo de transición a la L2}

Si bien en nuestro país nosotros propugnamos un modelo de mantenimiento para la educación bilinguie intercultural, y no uno de transición hacia la L2, hemos revisado también un modelo de este último 
tipo, el conocido como CALLA (Cognitive academic language learning approach). Éste se inicia con Chamot y O’Malley en 1994, y surge como un intento para resolver las dificultades académicas de los hijos de inmigrantes que aprendían inglés como segunda lengua en los Estados Unidos de Norteamérica.

Chamot y O’Malley señalan que "Los estudiantes de lenguas que están en minorías en éste [EEUU] y en otros países han encontrado, históricamente, dificultades en el aprendizaje de la lengua mayoritaria y en el rendimiento académico". ${ }^{1}$ (1994: 4) Entre las principales razones de estas dificultades en el aprendizaje, incluyen: la diferencia entre la cultura del niño y la cultura de la mayoría; fallas al tratar de promover el éxito inicial cognitivo y linguiístico en la lengua materna; y, un currículo, una instrucción y un desarrollo de personal inadecuados.

Los sujetos a los que va dirigido el enfoque CALLA son estudiantes de lenguas de minorías que han alcanzado un nivel de suficiencia en la segunda lengua, el inglés, correspondiente al grado más alto del nivel principiante, o a un nivel intermedio. Este modelo ofrece instrucción que les permite la transición, de un programa de aprendizaje de segunda lengua o de uno bilinguie, a los programas escolares regulares en donde se estudian todos los cursos en inglés. Si bien este modelo se articula sobre la base de tres componentes: temas, lengua académica, y estrategias de aprendizaje, por razones de nuestro tema de estudio, a continuación, haremos referencia sólo a los dos primeros

\subsubsection{Temas}

Los temas se toman de áreas de contenido del currículo de la escuela como Lenguaje, Matemática, Ciencia, y Estudios Sociales, contenidos que el alumno encontrará cuando se incorpore a las clases regulares en inglés a las que asisten los hablantes nativos de esta lengua.

Las áreas de contenido o cursos se introducen gradualmente, comenzando con la presentación de contenidos con un fuerte apoyo del contexto para el aprendizaje, o con un lenguaje que plantee poca exigencia. Así, el proceso se inicia con el curso de Ciencia porque un enfoque de descubrimiento y de tareas prácticas es compatible con un amplio apoyo contextual para el desarrollo del lenguaje académico. 
Además de que las experiencias pueden ser motivadoras para muchos estudiantes, esta práctica permite al alumno centrarse en el mensaje, y no en la lengua en sí. Un curso que puede ser introducido después es la Matemática, "que tiene su propio único lenguaje, especialmente en lo relativo a solucionar problemas con palabras, pero que, sin embargo, tiene menos exigencias de lengua que algunas de las otras áreas de contenido".2 (Chamot y O’Malley, 1994: 10) Luego se sugiere seguir con Estudios Sociales, aunque hay profesores que consideran que la geografía se puede enseñar más temprano por su lenguaje de exigencia reducida y su potencial relación con el conocimiento previo de los alumnos. Lenguaje iría en un cuarto lugar, aunque algunas personas argumentan a favor de introducir algunos aspectos, como cuentos con relevancia cultural, más temprano.

Un aspecto importante es la estimulación del desarrollo de habilidades de pensamiento exigentes por diferentes medios, controlando la exigencia que presenta la lengua para la actividad conceptual en áreas de contenido (1994: 10).

\subsubsection{Habilidades de lenguaje académico}

En cuanto al lenguaje, si bien se incluye el desarrollo de habilidades orales y escritas, estas se emplean como herramienta para el aprendizaje académico. No sólo se adquiere vocabulario y gramática del área de contenido, sino también conceptos y habilidades usando lenguaje académico. Además, se aprenden funciones para desempeñarse en el área de contenido como analizar, justificar, evaluar, y otras.

En resumen, los estudiantes desarrollan habilidades de lenguaje académico en la L2 a través de actividades con exigencia cognitiva, en las que la comprensión se apoya en soportes contextuales, y en las que se proporciona una instrucción con andamiaje adecuado como guía para adquirir el contenido.

\subsection{Programa de inglés intensivo en Lima}

Otra experiencia en la que se trata también temas de algunos cursos en la L2 es la de un colegio privado de clase media de Lima. Esta institución educativa no es un colegio bilingiie pues desarrolla todo el 
currículo en castellano respetándose no sólo la lengua materna sino también la cultura de los niños. Además de esto, se proporciona enseñanza de inglés como lengua extranjera durante diez horas semanales, en la primaria. Ocho de estas horas se dedican a la enseñanza aprendizaje de la lengua con materiales graduados que buscan desarrollar habilidades linguiísticas, tanto orales como escritas. En las otras dos horas, la institución viene desarrollando un proyecto con la L2 que se inició trabajando en quinto y sexto grados de primaria, planificándose práctica de lectura, con un material especial de ciencia en la segunda lengua. Debemos señalar que estos alumnos, aparte, hacen sus estudios de Ciencias Naturales en castellano.

Luego de la aplicación de la lectura en L2, se llegó a la conclusión de que se podía introducir esta práctica en grados más tempranos siempre que el profesor tuviera la calificación y experiencia necesarias para adecuarse al nivel de principiante de los alumnos. Se pudo constatar que los temas eran estimulantes para los niños y daban oportunidad para practicar la lengua, no por la lengua en sí, sino por el mensaje que ésta transmite, de manera similar a lo que sucede con la experiencia del modelo CALLA y del PEEB-Puno.. Por otro lado, el alumno también aprendió elementos básicos de lenguaje académico en la L2, demostrando que, como señala el PEEB-Puno, también de este modo se aprende algo de la L2.

Es importante precisar que aunque los textos que se emplean están ligeramente por encima del nivel de L2 de los alumnos, los temas y el diseño del material facilitan el trabajo del profesor. Junto con lecturas donde se explican conceptos de ciencia, se programa gran variedad de actividades y experimentos los que combinan lenguaje especialmente seleccionado e ilustraciones para guiar el aprendizaje. Los alumnos practican lengua centrándose en los temas, favoreciendo así la adquisición de aspectos de la segunda lengua. Veamos, a continuación, una actividad de lectura para el nivel de principiantes compuesto por la figura de una planta acompañada del siguiente texto. El tema es las partes de una planta.

What Parts Do Plants Have?

Plants have many parts.

Look at the picture.

It shows the parts of a plant. 
Color the flower yellow.

Color the stem and the leaves green.

Color the roots brown.

Hemos podido observar como un personal docente calificado en la especialidad de lengua extranjera desarrolla estrategias de enseñanza para adecuarse a los alumnos a su cargo. Las profesoras manifiestan que conviene comenzar con una actividad oral ligada a los conocimientos y experiencia previos de los niños. Así se les va preparando para lo nuevo que van a encontrar al acercarse al texto escrito. Algo fundamental es que el alumno realice actividades prácticas, manuales en lo posible, en el caso de los más pequeños.

De las observaciones de clase que hemos realizado, advertimos que se parte de una práctica de comunicación interpersonal, contextualizada, para luego pasar a una descontextualizada. Sin conocer la propuesta de Cummins, las profesoras experimentadas descubren lo mismo que este autor propone, la importancia del apoyo del contexto como preparación para el trabajo de lectura. De allí que se comience con la práctica oral antes mencionada, para después pasar al uso de los pasajes de lectura, en donde el alumno trabaja solo, con su libro.

\section{Conclusiones}

1. La enseñanza aprendizaje en segunda lengua no se puede desarrollar de la misma manera que en la lengua materna cuando los alumnos no tienen un dominio igual de ambas lenguas. Para el inicio del aprendizaje en L2 se requiere un fuerte apoyo del contexto o una exigencia reducida de la lengua.

2. El usar la lengua para estudiar contenidos de alguna área del currículo permite al estudiante el desarrollo del lenguaje académico de esa área haciéndolo adquirir elementos nuevos de la lengua o reforzar otros sin darse cuenta, de una manera comunicativa, pues el alumno está pensando en el mensaje, no en la lengua en sí. Esto supone un maestro conciente de lo que sucede con el aprendiz al procesar información, y conocedor de maneras para graduar la complejidad que puede ofrecer la lengua en el aprendizaje.

3. Como vemos en las tres experiencias, no es necesario tener un nivel avanzado en la L2 para iniciar el aprendizaje de contenidos del currí- 
culo en dicha lengua. Pero sí coinciden en que el alumno debe tener una base de la segunda lengua. De este modo, se puede realizar paralelamente la continuación del aprendizaje de la L2 y el aprendizaje de contenidos del currículo en la L2.

4. Desde el punto de vista del tiempo de adquisición de la lengua, los investigadores señalan una diferencia entre el desarrollo de lenguaje social, cotidiano, y el desarrollo de lenguaje académico. Falta hacer investigación en distintos contextos de nuestro país para ver cuánto tiempo se necesitaría para lograr el desarrollo de un lenguaje académico razonable.

5. La propuesta es introducir el trabajo con áreas de contenido gradualmente, esto es, no comenzar con varias asignaturas a la vez. Ninguno de los tres programas estudiado comienza con todos las áreas a la vez. Lo que sí se da en todos los casos es que los temas que se desarrollan son tomados del currículo regular.

Téngase en cuenta que las conclusiones a las que hemos llegado, tienen como base experiencias de distintas lenguas En este caso nos hemos referido al castellano como segunda lengua, pero consideramos que allgunas de estas ideas pueden ser útiles para cualquier otra lengua tratada como L2.

Notas

1. Traducción por M. Lozada.

2. Traducción por M. Lozada. 


\section{Referencias bibliográficas}

BAKER, Colin (1997) [1993]. Fundamentos de educación bilingüe y bilingüismo. Madrid: Ediciones Cátedra.

CHAMOT, Anna Uhl y O 'MALLEY, J. Michael (1994). The CALLA Handbook. USA: Addison-Wesley Publishing Company.

CEP ROSA DE AMÉRICA (2007). Informe sobre proyecto de lectura en L2. Documento interno de la institución.

CUMMINS, J. (2002). Lenguaje, poder y pedagogía. Madrid: Ministerio de Educación, Cultura y Deporte y Ediciones Morata.

CUMMINS, Jim, y Merrill SWAIN, (1986). Bilingualism in Education. New York: Longman.

MINISTERIO DE EDUCACIÓN PÚBLICA (2005). Enseñanza del Castellano como Segunda Lengua en las escuelas EBI del Perú. Manual para docentes de educación bilinguie intercultural. Lima: Dirección Nacional de Educación Bilinguie Intercultural.

MINISTERIO DE EDUCACIÓN PÚBLICA (1989): Jakawisata. Guía metodológica para el desarrollo de la asignatura de Ciencias Naturales en el 6to grado de Educación Primaria Bilinguie. Producción del Proyecto Experimental de Educación Bilinguie, Casilla 123, Puno. Lima-Puno.

SERIE FOCUS ON SCIENCE (2004): Student's Book - Levels: A, B, C, and D; Teacher's Guide: Levels A, B, C, and D. Texas: Harcourt Achieve, Austin. 